\title{
Tetrofosmin early time gated post-stress single-photon emission computed tomography imaging: Feasibility and potential benefits
}

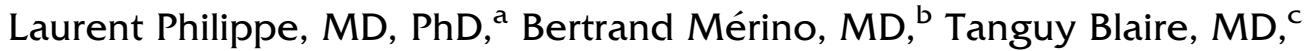 \\ Alban Bailliez, MD, ${ }^{d}$ Danielle Casset-Senon, PhD, ${ }^{e}$ Maurice Levy, $M D,{ }^{f}$ \\ Arnaud Halley, $M D,{ }^{\mathrm{g}}$ and Guillaume Divry, $\mathrm{MD}^{\mathrm{h}}$
}

Background. The purpose of this study is to evaluate the feasibility, the image quality, and the clinical relevance of an early gated post-stress (GPS) single-photon emission computed tomography (SPECT) tetrofosmin (Myoview ${ }^{\mathrm{TM}}$ - GE Healthcare) acquisition protocol. Time delay between myocardial technetium-labeled tracer administration and SPECT acquisition is usually about 30 minutes after stress, and 45 to 60 minutes at rest: because of the absence of significant redistribution, perfusion images are related to stress even 30 minutes after stress injection, while function and thickening data obtained with gated acquisition 30 minutes after stress are mainly related to rest conditions.

Methods. 194 patients were prospectively included and analyzed, in a multicenter registry. Three gated-SPECT ${ }^{9^{\mathrm{m}}}$ Tc-Tetrofosmin studies were performed per patient: GPS-SPECT, 30 minutes post-stress (GS30), and at rest (GR30).

Results. GPS image quality was excellent/good (93.9\%), and similar to GS30 images $(96.6 \%)$. The presence of adjacent myocardial sub-diaphragmatic activity on GPS images was similar to GS30 images (24\% vs $22 \%$ ), and less frequent than on GR30 images (31\%). For perfusion, thickening, and motion scores, there was no significant difference between early and 30 minute post-stress in the global patient population, but significant differences were observed between GPS and GS30 for LVEF $(65 \% \pm 15 \%$ vs $63 \% \pm 14 \%)$. In the ischemic patients, with the stress-rest protocol, the perfusion score was 14.2 on GPS images and 12.4 on GS30 images $(P=.002)$.

Conclusions. Tetrofosmin early GPS-SPECT is feasible without impairment of image quality (better count rate). Ischemic defect size on early post-stress images is slightly more pronounced than at 30 minutes: this could modify therapeutic decision. This technique produces reliable function information during early post-stress period, and might be useful for disclosing transient motion abnormalities. (J Nucl Cardiol 2011;18:62-72.)

Key Words: Tetrofosmin $\cdot$ myocardial perfusion imaging $\cdot$ gated SPECT $\bullet$ early imaging

From the Vinci Nuclear Medicine Center, ${ }^{\text {a }}$ Chambray-les-Tours, France; St Augustin Clinic, ${ }^{\mathrm{b}}$ Bordeaux, France; University Hospital Center, ${ }^{\mathrm{c}}$ Angers, France; Du Bois Clinic, ${ }^{\mathrm{d}}$ Lille, France; University Hospital Center, ${ }^{e}$ Chambray-les-Tours, France; HEP La Roseraie, ${ }^{f}$ Aubervilliers, France; GCS Nuclear Medicine, ${ }^{\mathrm{g}}$ Le Havre, France; and Nuclear Medicine Center, ${ }^{\mathrm{h}}$ Soissons, Amiens, France.

This study is conducted on behalf of the Multicenter Registry Group. Received for publication Oct 30, 2009; final revision accepted Sep 19, 2010.

Reprint requests: Laurent Philippe, MD, PhD, Vinci Nuclear Medicine Center, 9 Av. du Pr. Minkowski, 37170 Chambray-les-Tours, France; lphilippe@mednuc-tours.fr.

1071-3581/\$34.00

Copyright (C 2010 The Author(s). This article is published with open access at Springerlink.com

doi:10.1007/s12350-010-9302-7

\section{INTRODUCTION}

Myocardial single-photon emission computed tomography (SPECT) with technetium $99^{\mathrm{m}}$-labeled compounds allows electrocardiogram-gated acquisitions providing information on myocardial perfusion and left ventricular function.

Time delay between technetium-labeled tracer intravenous injection and data tomographic acquisition is usually about 30 minutes after stress, and 45 to 60 minutes after rest injection.

This time delay is usually equivalent to a rest function acquisition (when stunning is not present), even with stress tracer injection. 
Under usual conditions, the information obtained with gated tomography 45 to 60 minutes after stress injection is the following:

- perfusion imaging is related to stress perfusion (absence of significant redistribution),

- function and thickening information are related to rest LV function (stunning excepted).

By decreasing time delay between tracer injection and imaging, it is more likely that post-stress stunning in ischemic myocardium will be detected (the shorter the time-delay between stress injection and gated imaging, the maximum chance to detect ischemic wall motion abnormality). This information is of importance to demonstrate, for example, post-ischemic stunning, or to evaluate the LV stress adaptation by means of ejection fraction and volumes measurements, which are the main parameters for prognostic assessment.

After rest injection it has been suggested that it would be best to perform late imaging, whereas, after stress injection, in patients with suspected coronary artery disease, it seems possible, using $99 \mathrm{mTc}$-tetrofosmin, ${ }^{1}$ to start imaging very early post-stress injection. ${ }^{2}$ The purpose of this study is to evaluate the technical feasibility, the image quality, and the relevance of an early post-stress tetrofosmin Myoview $^{\mathrm{TM}}$ GE Healthcare)-gated acquisition protocol, and the potential benefits of this early acquisition.

\section{METHODS}

\section{Study Population}

We present the results obtained from 194 patients in a multicenter registry (eight centers). The registry was designed to collect data issued from normal daily clinical routine (suspicion or evaluation of coronary artery disease) studies and not to interfere with usual patient management. No additional examinations were requested. Oral information about the registry was given to all subjects, and written informed consent was obtained from each subject before the procedure. A total of 194 patients were enrolled: 134 men $(69 \%)$ and 60 women $(31 \%)$, mean age $62 \pm 10$ years, mean weight $76 \pm 14 \mathrm{~kg}$ (range $34-120 \mathrm{~kg}$ ) with a mean body mass index $27 \pm 4$ (range 14-40). Patient characteristics are described in Table 1; $80 \%$ of the patients presented with one or more coronary risk factors. A clinical history of coronary artery disease was present in 94 patients: a previous myocardial infarction in 39 , angina in 46 , previous revascularization with percutaneous coronary angioplasty or coronary artery bypass graft in 62. A previous exercise test (median age 3.0 months; range $0.1-180$ ), as part of the usual coronary patient management, has been performed in $51 \%$ of the patients (34\% with a positive test, $38 \%$ with a negative test, and $28 \%$ with an inconclusive test).
Table 1. Patients characteristics $(n=194)$

\begin{tabular}{lr}
\hline Mean age (years) (SD) & $62(10)$ \\
Male (\%) & $134(69 \%)$ \\
Female (\%) & $60(31 \%)$ \\
BMI $>27 \mathrm{~kg} / \mathrm{m}^{2}(\%)$ & $79(41 \%)$ \\
Risk factor for CAD (\%) & \\
No & $37(19 \%)$ \\
Diabetes mellitus & $42(22 \%)$ \\
Hypertension & $99(51 \%)$ \\
Dyslipidemia & $78(40 \%)$ \\
Positive family history & $67(35 \%)$ \\
History of CAD (\%) & \\
No & $100(52 \%)$ \\
Angina & $46(24 \%)$ \\
Myocardial infarction & $39(20 \%)$ \\
PCI or CABG & $62(32 \%)$ \\
Medications (\%) & \\
No* & $138(71 \%)$ \\
$\beta$-blocker & $36(19 \%)$ \\
Calcium antagonist & $19(10 \%)$ \\
Nitrates & $10(5 \%)$ \\
Vasodilator & $17(9 \%)$
\end{tabular}

No reliable data available on smoking.

$S D$, Standard deviation; $B M I$, body mass index; $C A D$, coronary artery disease; $P C I$, percutaneous coronary intervention; $C A B G$, coronary artery bypass graft surgery.

${ }^{*}$ At the time of the study.

\section{SPECT Procedure}

Three gated SPECT $99^{\mathrm{m}} \mathrm{Tc}$-Tetrofosmin were performed for each patient: early gated post-stress (GPS) SPECT, gated post-stress SPECT at 30 minutes (GS30), and gated rest SPECT at 30 minute post-IV injection (GR30). These three SPECT studies were acquired in a 1 day procedure. Stress-rest (120 patients) or rest-stress procedures (74 patients) were performed. Stress consisted of exercise (73\% of the patients), dipyridamole $(0.50$ to $0.65 \mathrm{mg} / \mathrm{kg}$ in 4 minutes) coupled with sub maximal exercise $(20 \%)$, and dipyridamole only $(0.50$ to $0.65 \mathrm{mg} / \mathrm{kg}$ in 4 minutes) $(7 \%)$.

Data were acquired on double-head gamma-cameras equipped with low energy high-resolution collimators. The protocol included $64 \times 64$ matrix, with zoom acquisition providing a pixel size of 6 to $7 \mathrm{~mm}$ depending on the gammacamera trademark, 32 projections over $180^{\circ}$ (L-mode, from RAO to LPO), and 16 gated frames per cardiac cycle. For the stress-rest protocol, the time projection acquisition durations were 50 seconds after stress and 30 seconds at rest. For the rest-stress protocol, projections were 50 seconds at rest, and 30 seconds after stress.

Tetrofosmin doses varied depending on patient weight. According to the European procedural guidelines, ${ }^{3}$ the administered activity of the second injection was three times the first injected activity. According to the summary of product characteristics, the total recommended dose, for gated studies 
should be of the order of 1,200 MBq (maximum). In our study, median total injected activity per patient was $1,136 \mathrm{MBq}$. Early SPECT GPS data were acquired after tetrofosmin injection as soon as possible after the stress test, $30 \pm 3$ minutes after tetrofosmin injection for GS30 data, and $31 \pm 6$ minutes after tetrofosmin rest injection for GR30 data. Time delay between stress and rest injections was $207 \pm 39$ minutes.

\section{Scintigraphic Data Analysis}

Computations of activity ratio (myocardium/liver and myocardium/lung) were obtained, for each patient and for each acquisition, by means of regions of interest (ROI) applied on a single-frame anterior projection: identical ROI size were applied for all patients (circular ROI, 10 pixels radius).

A semi-quantitative visual assessment of image quality was performed using a 4-point scale (from $0=$ excellent, to $3=$ poor). This assessment was performed in a similar way as in the Giogetti study. ${ }^{4}$ In order to estimate the image quality score, the following criteria were used: for score 0 (excellent quality), clinical imaging interpretation was reliable and easy; for a score of 1 (good), interpretation was also reliable but less easy; for a score of 2 (fair) quality interpretation was still suitable but more difficult, and for a score of 3 (poor), there was no reliable clinical image interpretation.

The presence (yes or no) of liver and gastro-intestinal activity adjacent to the myocardium (at least on one slice) was visually evaluated: sub-diaphragmatic activity equal or superior to adjacent myocardial activity was considered as disturbing. The reliability of endocardial/epicardial edge detection was assessed visually, mainly dependent on the presence or the absence of this splanchnic activity.

SPECT quantification has been computed using QPS ${ }^{\mathrm{TM}}$ and QGS ${ }^{\mathrm{TM}}$ software with the 20-segment system ${ }^{5}$ : each segment is scored ${ }^{6-8}$ using, for the perfusion, a 5-point system $(0=$ normal uptake to $4=$ absence of radioactive uptake). For wall motion semi quantitative assessment, a 6-point system is used ( $0=$ normal to $5=$ dyskinesis), and for thickening a four-point system $(0=$ normal to $3=$ no thickening). Summed scores were computed by adding the 20 segmental scores. Summed stress score (SSS), summed rest score, and summed difference score (SDS) were automatically calculated (with $\mathrm{QGS}^{\mathrm{TM}}$ software) for uptake, thickening, and motion analysis images. A normal sestamibi database (Cedars-Sinai Medical Center Normal limits) was used by the QPS software for tetrofosmin perfusion score analysis. This method has been used, for tetrosfosmin, with success in previous study. ${ }^{9}$

When the automated determination of the LV was not optimal or inappropriate, due to wrong edge detection, mainly in the basal region, an optional manual interaction was applied (mask and constrain). Eventually, if any of the segmental scores was deemed inaccurate, a manual correction was feasible after visual analysis, by left or right clicking on the inaccurate segment in the score box.

A SSS of 0 to 4 was considered as normal. ${ }^{10}$ Left ventricular ejection fraction (LVEF), end-diastolic volumes (EDV), and end-systolic volumes (ESV) were automatically computed by the QGS software.
Some parameters were not available (mainly the scores of 20 patients emanating from one participating center). Furthermore, when patient images where inadequate for some parameters, even after manual correction of the image processing, these values were excluded. The numbers of patients available for each parameter analysis are indicated in Table $2 \mathrm{~b}$.

\section{Statistical Analysis}

Continues variables are presented as mean and SD. The chi-square or Fisher tests were used for qualitative variables between unpaired groups, and ANOVA for quantitative variables (E3s Company-SAS v9.1 software). Comparison of quantitative variables between the different protocols were analyzed with paired Student's $t$ test (with Bonferroni correction for multiples paired comparisons), and McNemar's chisquare test for proportions comparisons. Correlation between two paired groups was assessed with Pearson coefficient and by simple least-square linear regression. A $P$ value $<.05$ was considered statistically significant.

\section{RESULTS}

\section{Stress Test}

The maximal heart rate was $91 \pm 10 \%$ for the exercise test, and $75 \pm 12 \%$ for the dipyridamole/exercise test. The stress test was electrocardiographically negative in 123 patients $(65 \%)$.

\section{Imaging Quality}

GPS image quality was excellent or good in $93.9 \%$ of cases, similar to GS30 images (96.6\%). Tracer activity in the liver and the gastrointestinal tract adjacent to the myocardium was present in $24 \%$ of the GPS images, $22 \%$ of the GS30 images, and $31 \%$ of the GR30 images (Table 2a).

The endocardial/epicardial border detection was considered as suitable for $92 \%$ of the GPS images, $93.7 \%$ for the GS30 images, and $89.5 \%$ for the GR30 images. The edge detection reliability varied, according to the stress-rest or rest-stress protocol (Table 3). The automated LV edge detection was more successful (Table 3) with the GPS stress-rest images (94\%) than with the GS30 stress-rest images $(91 \%)(P<.001)$. For the global population, myoc/liver and myoc/lung counts ratios were the following: myoc/liver: 0.94 (GPS), 1.21 (GS30), 0.91 (GR30) and myoc/lung: 2.72 (GPS), 2.92 (GS30), 2.52 (GR30). According to the protocol, the values are described in Table 3. For the normal patients, in accordance to the protocol, GPS, GS30, and GR30 myoc/liver and myoc/lung ratios are displayed in Table 4. In the normal patients sample, the average myocardial, lung, and liver ROI counts (79 pixels) were 
Table 2. Global population $(n=194)$-imaging quality

\begin{tabular}{|c|c|c|c|}
\hline & GPS & GS30 & GR30 \\
\hline \multicolumn{4}{|l|}{ (a) GPS image quality } \\
\hline \multicolumn{4}{|l|}{ Score quality (\%) } \\
\hline 0 & $130(87 \%)$ & $131(88 \%)$ & $129(87 \%)$ \\
\hline 1 & $10(7 \%)$ & $13(9 \%)$ & $15(10 \%)$ \\
\hline 2 & $8(5 \%)$ & $5(3 \%)$ & $4(3 \%)$ \\
\hline 3 & $1(1 \%)$ & $0(0 \%)$ & $0(0 \%)$ \\
\hline Mean heart/liver ratio (SD) & $0.94(0.33)$ & $1.21(0.44)$ & $0.91(0.36)$ \\
\hline Range heart/liver ratio & {$[0.35 ; 2.58]$} & {$[0.13 ; 2.93]$} & {$[0.34 ; 2.22]$} \\
\hline Mean heart/lung ratio (SD) & $2.72(0.70)$ & $2.92(1.06)$ & $2.52(0.52)$ \\
\hline Range heart/lung ratio & {$[0.79 ; 5.10]$} & {$[1.06 ; 13.79]$} & {$[1.29 ; 4.16]$} \\
\hline Splanchnic activity (\%) & $42(24 \%)$ & $38(22 \%)$ & $52(31 \%)$ \\
\hline Edge detection reliability (\%) & $161(92 \%)$ & $164(94 \%)$ & $154(90 \%)$ \\
\hline Mean perfusion score (SD) & $5.1(8.4)$ & $5.0(8.5)$ & $3.5(7.0)$ \\
\hline Range perfusion scores & {$[0 ; 56]$} & {$[0 ; 57]$} & {$[0 ; 48]$} \\
\hline Mean thickening score (SD) & $4.2(6.9)$ & $4.3(6.5)$ & $3.7(5.7)$ \\
\hline Range thickening scores & {$[0 ; 37]$} & {$[0 ; 38]$} & {$[0 ; 33]$} \\
\hline Mean motion score (SD) & $4.5(8.6)$ & $4.8(8.2)$ & $4.2(7.7)$ \\
\hline Range motion scores & {$[0 ; 48]$} & {$[0 ; 46]$} & {$[0 ; 44]$} \\
\hline \multicolumn{4}{|l|}{ (b) Number of patients available } \\
\hline Score quality $(\mathrm{n})$ & 149 & 148 & 146 \\
\hline Mean heart/liver ratio (n) & 191 & 191 & 190 \\
\hline Mean heart/lung ratio $(n)$ & 191 & 191 & 189 \\
\hline Splanchnic activity (n) & 179 & 174 & 167 \\
\hline Edge detection reliability (n) & 175 & 175 & 172 \\
\hline Mean perfusion score $(\mathrm{n})$ & 174 & 174 & 172 \\
\hline Mean thickening score $(\mathrm{n})$ & 162 & 167 & 161 \\
\hline \multirow[t]{2}{*}{ Mean motion score $(\mathrm{n})$} & 149 & 150 & 145 \\
\hline & $\begin{array}{c}\text { GPS-GS30 } \\
\text { (P value) }\end{array}$ & $\begin{array}{c}\text { GPS-GR30 } \\
\text { (P value) }\end{array}$ & $\begin{array}{c}\text { GS30-GR30 } \\
\text { (P value) }\end{array}$ \\
\hline \multicolumn{4}{|c|}{ (c) Comparisons of GPS-GS30, GPS-GR30, and GS30-GR30 } \\
\hline Mean heart/liver ratio & $<.001$ & NS & $<.001$ \\
\hline Mean heart/lung ratio & $<.003$ & $<.001$ & $<.001$ \\
\hline Mean perfusion score & NS & $<.001$ & $<.001$ \\
\hline
\end{tabular}

Score quality: $0=$ excellent; $1=$ good; $2=$ fair; $3=$ poor.

GPS-GS30, GPS-GR30, and GS30-GR30: comparisons of two protocols by paired Student's $t$ test with Bonferroni correction for multiple (3) comparisons.

GPS, Early gated post-stress; GS30, gated 30 minute post-stress; GR30, gated 30 minute rest; $S D$, standard deviation; $n$, number of patients suitable for each analysed parameter; NS, nonsignificant.

6925 (88/pix.), 2410 (30/pix.), and 10470 (133/pix.), respectively, for the GPS images. For the GS30 images, counts were 5810 (74/pix.), 1940 (23/pix.), and 7450 (94/pix.), respectively, and for the GR30 images, these values were 12240 (155/pix.), 4490 (57/pix.), and 18245 (231/pix.).

The worst results were observed with the rest-stress protocol for the GR30 images (only 77\% of the images were suitable).
The decrease of myocardial counts rate between GPS and GS30 images is evaluated to $12 \%$ (after $4 \%$ radioactive decay correction for 20-25 minute interval).

\section{Perfusion Imaging and Myocardial Function Analysis}

Considering the total patient population, there was no significant difference for the perfusion scores (SSS) 
Table 3. Global population-imaging quality, according to the protocol (stress-rest or rest-stress)

\begin{tabular}{|c|c|c|c|c|c|c|}
\hline \multirow[b]{2}{*}{ Protocol } & \multicolumn{2}{|c|}{ GPS } & \multicolumn{2}{|c|}{ GS30 } & \multicolumn{2}{|c|}{ GR30 } \\
\hline & $\begin{array}{c}\text { S-R } \\
(n=120)\end{array}$ & $\begin{array}{c}\text { R-S } \\
(n=74)\end{array}$ & $\begin{array}{c}\text { S-R } \\
(n=120)\end{array}$ & $\begin{array}{c}\text { R-S } \\
(n=74)\end{array}$ & $\begin{array}{c}\text { S-R } \\
(n=120)\end{array}$ & $\begin{array}{c}\text { R-S } \\
(n=74)\end{array}$ \\
\hline Mean heart/liver ratio (SD) & $0.96(0.37)$ & $0.91(0.27)$ & $1.14(0.41)$ & $1.33(0.47)$ & $1.00(0.36)$ & $0.76(0.286)$ \\
\hline$P$ value (S-R vs $\mathrm{R}-\mathrm{S}$ ) & .272 & & $.004^{*}$ & & $<.001^{*}$ & \\
\hline Mean heart/lung ratio (SD) & $2.83(0.78)$ & $2.54(0.49)$ & $2.99(0.79)$ & $2.82(1.39)$ & $2.64(0.51)$ & $2.33(0.48)$ \\
\hline$P$ value (S-R vs $\mathrm{R}-\mathrm{S}$ ) & $.004^{*}$ & & .269 & & $<.001^{*}$ & \\
\hline Splanchnic activity n (\%) & $32(31 \%)$ & $10(14 \%)$ & $28(28 \%)$ & $10(14 \%)$ & $23(25 \%)$ & 29 (39\%) \\
\hline$P$ value (S-R vs $\mathrm{R}-\mathrm{S}$ ) & $.012^{*}$ & & $.026^{*}$ & & .064 & \\
\hline $\begin{array}{l}\text { Edge detection } \\
\text { reliability n (\%) }\end{array}$ & 95 (94\%) & $66(89 \%)$ & $92(91 \%)$ & $72(97 \%)$ & 97 (99\%) & $57(77 \%)$ \\
\hline$P$ value (S-R vs $\mathrm{R}-\mathrm{S}$ ) & .269 & & .121 & & $<.001 *$ & \\
\hline$P$ value (S-R: GPS vs GS30) & $<.001$ & & & & & \\
\hline Mean heart/liver ratio (n) & 117 & 74 & 117 & 74 & 116 & 74 \\
\hline Mean heart/lung ratio (n) & 117 & 74 & 117 & 74 & 115 & 74 \\
\hline Splanchnic activity & 102 & 74 & 100 & 74 & 93 & 74 \\
\hline Edge detection reliability (n) & 101 & 74 & 101 & 74 & 101 & 74 \\
\hline
\end{tabular}

GPS, Early gated post-stress; GS30, gated 30 minute post-stress; GR3O, gated 30 minute rest; $S D$, standard deviation; $S-R$, stressrest; $R$-S, rest-stress; $n$, number of patients suitable for each analysed parameter.

${ }^{*} P<.05-$ nonparametric Fisher test, for unpaired series; McNemar test for proportion comparison of paired series.

early and at 30 minute post-stress, whatever the protocol (Tables 2a-c). The linear regression between SSS early and at 30 minute post-stress, for the stress-rest protocol SSS GPS was $0.63+0.94$ SSS GR30 (Pearson correlation coefficient $=0.97 P<.001$ ), and for the reststress protocol SSS GPS was $0.09+0.96$ SSS GR30 (Pearson correlation coefficient $=0.94 P<.001$ ) .

There is also no significant difference for the thickening scores, and for the motion scores, early and at 30 minute post-stress, whatever the protocol.

The volumes (EDV and ESV) were in the same range for the different acquisitions, without significant difference between GPS, GR30, and GR30, whatever the protocol. Whereas GPS LVEF was slightly greater than GS30 and GR30 LVEF $(P<.001$ and $P<.006$, respectively) (Table 5).

\section{Sub-Group Analysis: Normal Perfusion Versus Abnormal Perfusion}

Normal patients were defined with a SSS $<4$ on GPS images. Thus, $40 \%$ of the patients were considered as abnormal (SSS $\geq 4$ ). Among these patients, 33\% presented with fixed defects, and $67 \%$ had reversible abnormalities.

In the normal group, there was no significant difference between the three SPECT acquisitions for some parameters (thickening, motion, and volumes) listed in Table 6. In this normal patient group, the statistical differences observed related to the perfusion scores of GPS vs GS30, GS30 vs GR30 $(P<.001$ and $P<.001$, respectively), and LVEF GPS vs GS30 $(P<.001)$.

Whereas, in the abnormal perfusion group, we observe different results depending on the protocol and on the kind of defect, in the ischemic patients, the only statistical significant difference was observed for the perfusion score between GPS images and GS30 images for the stress-rest protocol. The SSS was 14.2 on early GPS images and 12.4 on 30 minute post-stress images $(P=.002)$. The SDS was 6.0 with early GPS images and 4.2 with the 30 minute post-stress images (Table 7). The linear regression between GPS SSS and GS30 SSS, for ischemic patients employing the stress-rest protocol, was GPS SSS $=3.46+0.854$ GS30 SSS, with a 0.946 Pearson correlation coefficient $(P<.001)$. The GPS SSS early post-stress regression-predicted values are greater than the 30 minute post-stress GS30 SSS up to GS30 of 24. Beyond this value, both SSS are close to the identity line (Figure 1). The linear correlation offset (3.46) confirms the trend toward larger GPS SSS (vs GS30 SSS) for the small defects.

For the others parameters, we did not find any significant difference between the three SPECT data sets, either for the fixed defects or for the ischemic patients. 
Table 4. Normal patients-imaging quality, according to the protocol (stress-rest or rest-stress)

\begin{tabular}{|c|c|c|c|c|c|c|}
\hline \multirow[b]{2}{*}{ Protocol } & \multicolumn{2}{|c|}{ GPS } & \multicolumn{2}{|c|}{ GS30 } & \multicolumn{2}{|c|}{ GR30 } \\
\hline & $\begin{array}{c}\text { S-R } \\
(n=49)\end{array}$ & $\begin{array}{c}\text { R-S } \\
(n=55)\end{array}$ & $\begin{array}{c}\text { S-R } \\
(n=49)\end{array}$ & $\begin{array}{c}\text { R-S } \\
(n=55)\end{array}$ & $\begin{array}{c}\text { S-R } \\
(n=49)\end{array}$ & $\begin{array}{c}\text { R-S } \\
(n=55)\end{array}$ \\
\hline Mean heart/liver ratio (SD) & $0.93(0.30)$ & $0.92(0.25)$ & $1.11(0.37)$ & $1.33(0.44)$ & $0.95(0.33)$ & $0.77(0.26)$ \\
\hline$P$ value (S-R vs $\mathrm{R}-\mathrm{S})$ & .973 & & $.009^{*}$ & & $<.003 *$ & \\
\hline Mean heart/lung ratio (SD) & $2.86(0.74)$ & $2.57(0.42)$ & $3.03(0.74)$ & $2.94(1.56)$ & $2.66(0.53)$ & $2.38(0.47)$ \\
\hline$P$ value (S-R vs $\mathrm{R}-\mathrm{S}$ ) & $.013^{*}$ & & .713 & & $<.005^{*}$ & \\
\hline Splanchnic activity n (\%) & $9(20 \%)$ & $7(13 \%)$ & $11(24 \%)$ & $7(13 \%)$ & $9(20 \%)$ & $19(35 \%)$ \\
\hline$P$ value (S-R vs R-S) & .417 & & .190 & & .122 & \\
\hline Edge detection reliability $n(\%)$ & $46(96 \%)$ & $48(87 \%)$ & $46(94 \%)$ & $53(96 \%)$ & $46(98 \%)$ & $44(80 \%)$ \\
\hline$P$ value (S-R vs $\mathrm{R}-\mathrm{S})$ & .170 & & .665 & & $<.005^{*}$ & \\
\hline Mean heart/liver ratio $(\mathrm{n})$ & 48 & 55 & 48 & 55 & 47 & 55 \\
\hline Mean heart/lung ratio $(\mathrm{n})$ & 48 & 55 & 48 & 55 & 46 & 55 \\
\hline Splanchnic activity (n) & 46 & 55 & 45 & 55 & 45 & 55 \\
\hline Edge detection reliability (n) & 48 & 55 & 49 & 55 & 47 & 55 \\
\hline
\end{tabular}

GPS, Early gated post-stress; GS3O, gated 30 minute post-stress; GR3O, gated 30 minute rest; $S D$, standard deviation; $S-R$, stressrest; $R$-S, rest-stress; $n$, number of patients suitable for each analysed parameter.

${ }^{*} P<0.05-$ nonparametric Fisher test for unpaired series.

Table 5. Global population $(n=194)$ : ejection fraction, and left ventricular volumes

\begin{tabular}{|c|c|c|c|c|c|c|}
\hline & GPS & GS30 & GR30 & $\begin{array}{c}\text { GPS-GS30 } \\
\text { (P value) }\end{array}$ & $\begin{array}{c}\text { GPS-GR30 } \\
\text { ( } P \text { value) }\end{array}$ & $\begin{array}{c}\text { GS30-GR30 } \\
\text { (P value) }\end{array}$ \\
\hline Mean LVEF (\%) (SD) & $65(15)$ & $63(14)$ & $63(13)$ & $<.001$ & $<.006$ & NS \\
\hline Range LVEF (\%) & {$[12 ; 99]$} & {$[22 ; 100]$} & {$[23 ; 100]$} & & & \\
\hline Mean EDV (mL) (SD) & $85(44)$ & $87(36)$ & $90(38)$ & NS & NS & NS \\
\hline Range EDV $(\mathrm{mL})$ & {$[14 ; 453]$} & {$[21 ; 244]$} & {$[15 ; 292]$} & & & \\
\hline Mean ESV $(\mathrm{mL})(\mathrm{SD})$ & $34(38)$ & $36(27)$ & $36(28)$ & NS & NS & NS \\
\hline Range ESV $(\mathrm{mL})$ & [0; 397] & {$[0 ; 190]$} & {$[0 ; 226]$} & & & \\
\hline
\end{tabular}

$S P E C T$, Single photon emission computed tomography; $L V E F$, left ventricular ejection fraction; $E D V$, end diastolic volume; $E S V$, end systolic volume; GPS, early gated post-stress; GS3O, gated 30 minute post-stress; GR30, gated 30 minute rest; SD, standard deviation; NS, nonsignificant.

GPS-GS30, GPS-GR30, and GS30-GR30: comparisons of two protocols by paired Student's $t$ test with Bonferroni correction for multiple (3) comparisons.

Furthermore, we observed only one patient with strictly normal perfusion image at 30 minutes (GS30 SSS $=0$ ) and abnormal perfusion on GPS images (SSS > 3).

In the ischemic group, with the stress-rest protocol, there were no significant differences in LVEF between 10 and 30 minute post-stress, and at rest (respectively $57.4 \pm 14.1 \%, \quad 57.5 \pm 15.6 \%, \quad$ and $\quad 59.7 \pm 11.7 \%$ ) although we found some patients with transient stunning.

Among patients $(\mathrm{n}=10 / 27)$ with GPS LVEF $<$ GR30 LVEF (with a 5\% minimum difference), only 2 patients presented a GPS LVEF $<$ GS30 LVEF.

\section{DISCUSSION}

\section{Feasibility of Early Time GPS SPECT, and Reliability of GPS Information}

We confirm the feasibility of tetrofosmin early time gated post-stress SPECT. According to Jain et al, ${ }^{2}$ tetrofosmin heart imaging can be started as soon as 5 minutes after stress-injection. In the publication of Giorgetti et $\mathrm{al}^{4}{ }^{4} 15$ minute tetrofosmin post-stress SPECT images were suitable for qualitative clinical interpretation. According to Costo et al, ${ }^{11}$ early (10 
Table 6. Normal patients $(\mathrm{n}=104)$ : scores and LV functional parameters

\begin{tabular}{|c|c|c|c|c|c|c|}
\hline & GPS & GS30 & GR30 & $\begin{array}{c}\text { GPS-GS30 } \\
\text { (P value) }\end{array}$ & $\begin{array}{c}\text { GPS-GR30 } \\
\text { (P value) }\end{array}$ & $\begin{array}{c}\text { GS30-GR30 } \\
\text { (P value) }\end{array}$ \\
\hline Mean perfusion score (SD) & $0.5(0.9)$ & $0.9(1.6)$ & $0.3(1.1)$ & $<.001$ & NS & $<.001$ \\
\hline Range perfusion score & {$[0 ; 3]$} & {$[0 ; 8]$} & {$[0 ; 8]$} & & & \\
\hline Mean thickening score (SD) & $1.6(2.9)$ & $1.8(2.5)$ & $1.6(2.3)$ & NS & NS & NS \\
\hline Range thickening score & {$[0 ; 18]$} & {$[0 ; 13]$} & {$[0 ; 12]$} & & & \\
\hline Mean motion score (SD) & $2.3(5.3)$ & $2.5(4.0)$ & $2.1(4.2)$ & NS & NS & NS \\
\hline Range motion score & {$[0 ; 39]$} & {$[0 ; 22]$} & {$[0 ; 32]$} & & & \\
\hline Mean LVEF (\%) (SD) & $69(13)$ & $66(12)$ & $67(11)$ & $<.001$ & NS & NS \\
\hline Range LVEF (\%) & [12; 97] & {$[31 ; 100]$} & {$[41 ; 94]$} & & & \\
\hline Mean EDV (mL) (SD) & $78(45)$ & $78(27)$ & $80(27)$ & NS & NS & NS \\
\hline Range EDV $(\mathrm{mL})$ & {$[14 ; 453]$} & {$[21 ; 160]$} & {$[15 ; 141]$} & & & \\
\hline Mean ESV (mL) (SD) & $28(40)$ & $28(17)$ & $28(15)$ & NS & NS & NS \\
\hline Range ESV $(\mathrm{mL})$ & {$[1 ; 397]$} & [0; 93] & {$[1 ; 65]$} & & & \\
\hline
\end{tabular}

Abbreviations as in Tables 2, 3, and 4.

Table 7. Ischemic patients: scores differences for stress-rest and rest-stress protocols

\begin{tabular}{|c|c|c|c|c|}
\hline \multirow[b]{2}{*}{ Difference GPS-GS30 } & \multicolumn{2}{|c|}{ Stress-rest $(n=31)$} & \multicolumn{2}{|c|}{ Rest-stress $(n=16)$} \\
\hline & Descriptive statistics & $P$ value ${ }^{a}$ & Descriptive statistics & $P$ value ${ }^{a}$ \\
\hline Mean diff. perfusion score (SD) & $1.8(3.03)$ & $0.002^{*}$ & $1.2(3.10)$ & .147 \\
\hline Range perfusion scores & {$[-2 ; 9]$} & & {$[-5 ; 9]$} & \\
\hline Mean diff. thickening score (SD) & $-0.7(4.2)$ & 0.369 & $0.8(2.4)$ & .227 \\
\hline Range thickening scores & {$[-8 ; 12]$} & & {$[-3 ; 7]$} & \\
\hline Mean diff. motion score (SD) & $-0.9(6.8)$ & 0.555 & $-0.9(4.1)$ & .403 \\
\hline Range motion scores & {$[-13 ; 19]$} & & {$[-11 ; 10]$} & \\
\hline
\end{tabular}

SPECT, Single photon emission computed tomography; GPS-GS3O, difference of summed scores between early post-stress and 30 minute post-stress; $S D$, standard deviation.

* $P \leq .05$.

a Student paired $t$ test.

minutes) tetrofosmin gated post-stress is feasible, reproducible, and gives reliable function assessment. In our study, the SPECT performed just after stress was also of adequate quality for clinical interpretation. Guernou et al $^{12}$ suggested also that early post-stress (10 minutes) gated Sestamibi SPECT is feasible and provides adequate perfusion information.

The reliability of LV edge detection was as good for GPS images as for 30 minute post-stress images. Usually, when sub-diaphragmatic activity, close to the myocardium, is high on GPS images, it persists at 30 minutes, especially for post-dipyridamole images, even after cold water drinking and walking.

The poor GR30 image quality of the rest-stress protocol is probably related to the low dose activity and to the resting physiological conditions which does not favor the rest-stress protocol for reliable resting images.
Nevertheless, this fact does not relate to the quality of GPS images.

In order to detect ischemia with a rest-stress protocol, it is necessary to get a significant defect on the second set of images, over residual activity from the resting images: this is less easy than to detect a defect on the first set of images (stress) and to look for its disappearance on the second set (rest). The lower values of the heart/liver (nonsignificant) and heart/lung ratios $(P=.004)$ on GPS images for the rest-stress protocol when compared with the stress-rest protocol (Table 3) give evidence of this residual activity on stress images. This could be a reason explaining the difference in the results (Table 7), especially for small defects. Nevertheless, the good count rate of the high dose for the stress injection could partially compensate the previous drawback. 
PERFUSION scores - GPS vs. GS30 Ischemic patients - stress-rest protocol

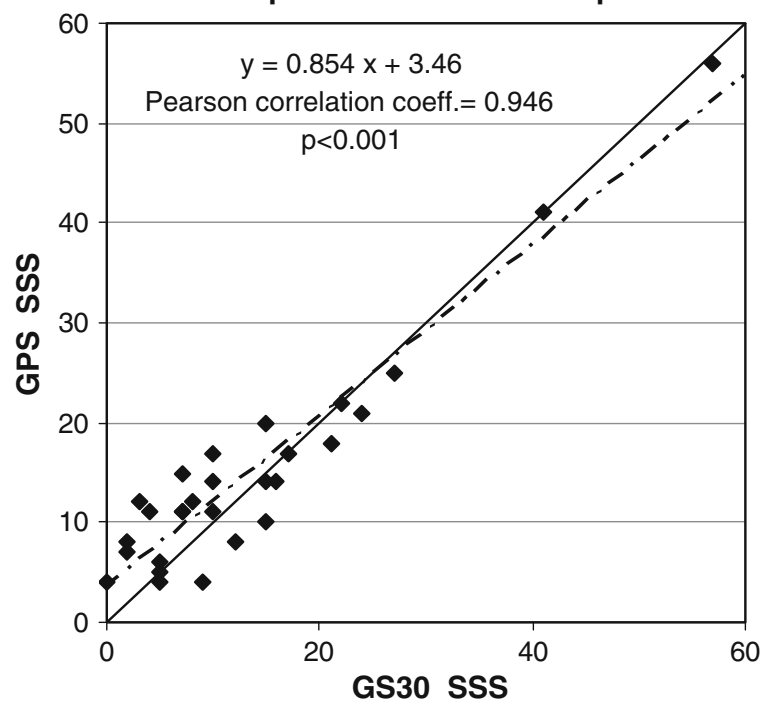

Figure 1. Relation between GPS SSS and GS30 SSS: The defect extent of the early post-stress (GPS SSS) is a little larger than on the 30 minute post-stress (GS30 SSS). The regression line (dashed line) predicts GPS values greater than GS30 values for GS30 SSS between 4 and 24, and close to the identity line (solid line) for GS30 values higher than 24 .

GPS heart/lung ratios were smaller than GS30 ratios $(P<.001$, Table 2$)$, mainly due to relative higher lung counts on GPS images. This pulmonary uptake can be of clinical value: for Georgoulias et al, ${ }^{13}$ early post-stress (4-6 minutes) pulmonary tetrofosmin uptake has incremental predictive value for coronary artery disease.

Acquiring early post-stress images, allows a $4 \%$ saving in counts rate when compared to 30 minute images post-stress, due to 20-25 minute radioactive decay. Furthermore, due to the myocardial clearance between 10 and 30 minutes, there is an additional $12 \%$ counts loss. ${ }^{2}$ This value agrees with our observed myocardial counts. Thus, early imaging avoids $16 \%$ waste of count rate.

\section{Comparison of Size of GPS Perfusion Defects Versus 30 Minute Imaging}

The only difference observed between early images and 30 minute post-stress images concerned the scoring of the perfusion defect for the ischemic patients evaluated with the stress-rest protocol: the extent and severity of the defect on early GPS data is slightly larger than at 30 minutes $(P=.002)$. Giorgetti et $\mathrm{al}^{4}$ observed a similar result in their study. They observed a more severe post-stress defect in early (15 minutes) than at 45 minute imaging. Costo et $\mathrm{al}^{11}$ also found that the defects were more severe at 10 minutes than at 30 minute post-stress.

The explanations of the larger extent and severity of the perfusion defect on the early post-stress images when compared with the 30 minute images are not well defined. As mentioned by Giorgetti et al, ${ }^{4}$ by acquiring earlier images, more ischemia is more likely to be observed. These authors attribute the early more obvious perfusion defect to the partial-volume effect because of the more pronounced wall thinning induced by the ischemia. Nevertheless, we do not think that this explanation alone can support this phenomenon. Indeed, we observed this difference of score between early images and 30 minute images for small defects, without obvious wall thinning and without consequence on epicardial/endocardial edge detection. In our series of ischemic patients, the SSS was larger on early images than at 30 minutes, but without wall motion abnormalities sufficient to generate a partial volume effect.

Another plausible explanation concerns the existence of partial redistribution with time, due to tracer washout. According to Schulz et al, ${ }^{14}$ there is a washout fraction per time lower in regions with severely reduced stress perfusion ( $8.3 \%$ per hour) than in normal $(11.5 \%$ per hour) segments or segments with moderately reduced perfusion (11.2\% per hour). This differential washout could partially explain why ischemic defects are smaller at 30 minutes than at 10 minutes: the slower washout maintains myocardial tissue activity at a relative constant level when compared to the normal area where the biological decay is more pronounced. This differential washout behavior involves a decrease with time of the contrast uptake between ischemic and normal area.

In our opinion, another mechanism could also participate to this phenomenon. The uptake at 10-15 minutes may be incomplete in area with mildly reduced coronary flow reserve. In low flow areas, the maximum uptake may be delayed. Thus, when early imaging is performed the activity in this abnormal region is lower than in the images acquired 20 minutes later. Probably this mechanism is valid only for mild defects, since for the normal area, the maximum uptake is reached quickly, and for severe defect area, the activity remains low whatever the time delay. Sinusas et $\mathrm{al}^{15}$ observed no significant differences in myocardial clearance between ischemic and nonischemic regions over the initial 15 minutes after injection, but it should be noted that the study concerned dogs with severe ischemia (complete left anterior descending coronary artery occlusion, followed by pharmacological stress). Ito et $\mathrm{al}^{16}$ have demonstrated a slight filling of $99^{\mathrm{m}} \mathrm{Tc}$-tetrofosmin in ischemic segments at approximately 1 hour on the exercise image. 
In the COURAGE study, ${ }^{17}$ patients with $\geq 10 \%$ ischemic myocardium were significantly improved with percutaneous coronary intervention: the assessment of the percent ischemic myocardium is of importance because of the therapeutic consequence. In our study, the SDS with early GPS images was 1.8 points greater than seen with 30 minute post-stress images. According to the ASNC imaging guidelines for nuclear cardiology procedures, ${ }^{18}$ the semi-quantitative scoring system may be used to define reversibility as a $\geq 2$-grade improvement, or improvement to a score of 1 . Then, a small increase of the SDS can change the report from a nonreversible to reversible defect. A small increase of percent ischemic myocardium, from $<10 \%$ (on 30 minute images) to $\geq 10 \%$, could modify the therapeutic strategy, and then the prognosis. In the COURAGE study, ${ }^{17}$ patients with $\geq 10 \%$ ischemic myocardium had ischemia reduced more with coronary revascularization than with medical therapy. This yielded a better event-free survival. In our study, among the 31 ischemic patients, 11 had a GS30 SSS $<8$ (equivalent to a $10 \%$ defect) but 6 patients in this group had a GPS score $>8$. Thus, in our study, 19\% (6/31) of the ischemic patients, according to the COURAGE study findings, would be treated by a more aggressive therapy. However, in the COURAGE Nuclear Sub Study, the percent ischemic myocardium was evaluated on 30 minute post-stress images, while, in our study, we evaluated the defect on early acquisition: then a new threshold should be determined for these new acquisition circumstances.

\section{Comparison of LV Function on GPS Versus 30 Minute Imaging}

The mean LVEF is quite similar on early versus 30 minute images, even for the ischemic group. Despite the more marked perfusion defect seen on early imaging, the number of patients with large ischemic defects is too small for getting statistically differences. Furthermore, the significant observed differences concerned LVEF higher on early imaging than at 30 minutes (Tables 5, 6). Small wall motion abnormalities can be compensated by a healthy myocardial area stress response, especially on early images, yielding effective higher post-stress LVEF.

\section{Stunning}

Emmet et $\mathrm{al}^{19}$ showed that stress-induced (i.e., reversible) wall motion abnormalities are highly specific for severe angiographic stenosis. Thus, delayed imaging (30 minutes) could miss these functional abnormalities. We observed, in few cases, wall motion abnormalities on early images that resolved at 30 minutes, consistent with transient myocardial stunning. Some authors think that these findings may not be related to myocardial stunning, but to incorrect endocardial edge detection in ischemic areas. ${ }^{20,21}$ Nevertheless, the wall abnormality is less obvious on delayed images, even when the perfusion defect is still present (may be less extended, but not sufficient to explain a false positive on early images, and an adequate endocardial edge detection on delayed imaging). This explanation is in agreement with several authors: for Toba et $\mathrm{al}^{22}$ imaging soon after exercise was superior to conventional late imaging to discriminate patients with single-vessel disease and postexercise stunning. Usually, it is considered that a 5\% decrease of stress LVEF in comparison with rest LVEF, associated with a reversible wall motion abnormality is consistent with myocardial stunning. Stunning can be observed after exercise stress, and rarely after dipyridamole test (coronary reserve test). For the detection of stunning by gated SPECT, regional dysfunction may be more sensitive than global dysfunction. ${ }^{23}$

\section{Study Limitations}

As a consequence of a multicenter registry, the studies were performed with different equipments. Nevertheless, data were acquired with similar gammacamera characteristics. Sensitivity and specificity of this early imaging protocol were not available, due to the frequent absence of coronary angiography. The study population included a large proportion of normal patients, derived from normal daily clinical imaging (suspicion or evaluation of coronary artery disease). The patients were mostly outpatients. Secondary to this, the wall motion abnormalities are small, and the LVEF is rather high. This may explain, in part, why the differences are not significant. The LVEF difference between early and 30 minute post-stress is related to a normal exercise myocardial response. For the ischemic patients, the possible LVEF decrease is masked by the normal area contraction response. Furthermore, the number of abnormal studies is small, and especially for the ischemic patients (47 out of 194).

Because we did not possess a tetrofosmin normal database, we used the sestamibi data included in the software, leading to a potential bias: Nevertheless, many studies demonstrate, between both tracers, similar distribution patterns, similar defect sizes, and no significant differences for the summed uptake scores. ${ }^{24,25}$

Another limitation concerns the use of a 20 -segment model for myocardial analysis, since the 17-segment model is now preferred (our choice was due to the software availability in the different centers). Berman et $\mathrm{al}^{26}$ showed in their study that the 17 -segment model 
demonstrated a trend toward fewer mildly abnormal scans and more normal and severe abnormal scans. So it is possible that with a 17 -segment model, the number of patients with abnormal scan will decrease. According to Slomka, ${ }^{10}$ segmental scores are rounded off to integer values, and use coarse segment boundaries. A small defect located between two segments may be underestimated.

\section{CONCLUSION}

Myoview early time gated post-stress SPECT is feasible without impairment of image quality (better count rate). This technique also produces reliable function information during early post-stress period, as reliable as those obtained at 30 minute post-stress. The first benefit of this protocol is logistic in that it is not necessary to wait between the stress test and the SPECT acquisition.

Another benefit concerns the assessment of the extent and severity of the ischemic defects (for the stress-rest protocol): these defects could be slightly more obvious early post-stress than at 30 minutes, providing a better sensitivity. Disclosing more pronounced perfusion and function abnormalities, using early post-stress acquisitions, could influence the therapeutic modalities and therefore the patient prognosis. A small additional decrease of LVEF between early and 30 minute acquisitions could produce a modification of the final report from normal to transient myocardial stunning.

Finally, we think that this early protocol is at least as efficient as the conventional 30 minute post-stress acquisition, more convenient to organize, and potentially more informative.

\section{Acknowledgments}

We would like to thank Alain Chetanneau, M.D., Philippe Pezard, M.D., Sylvie Petit, M.D., Frédéric Patrois, M.D., Olivier Sonnet, M.D., Cyrille Blondet, M.D., and Laurent Macé, M.D., for their contribution to this study. The authors appreciated the quality of the Journal reviewers' comments and thank them for their detailed analysis of the manuscript.

\section{Conflicts of interest}

The authors have indicated they have no financial conflicts of interest.

\section{Open Access}

This article is distributed under the terms of the Creative Commons Attribution Noncommercial License which permits any noncommercial use, distribution, and reproduction in any medium, provided the original author(s) and source are credited.

\section{References}

1. Matsunari I, Tanishima Y, Taki J, et al. Early and delayed technetium-99m-tetrofosmin myocardial SPECT compared in normal volunteers. J Nucl Med 1996;37:1622-6.

2. Jain D, Wackers FJTh, Mattera J, McMahon M, Sinusas AJ, Zaret BL. Biokinetics of technetium-99m-tetrofosmin: Myocardial perfusion imaging agent: Implications for a one-day imaging protocol. J Nucl Med 1993;34:1254-9.

3. Hesse B, Tägil K, Cuocolo A, et al. EANM/ESC procedural guidelines for myocardial perfusion imaging in nuclear cardiology. Eur J Nucl Med Mol Imaging 2005;32:855-97.

4. Giorgetti A, Rossi M, Stanislao M, et al. Feasibility and diagnostic accuracy of a gated SPECT early-imaging protocol: A multicenter study of the myoview imaging olptimization group. J Nucl Med 2007;48:1670-5.

5. Cerqueira MD, Weissman NJ, Dilsizian V, et al. Standardized myocardial segmentation and nomenclature for tomographic imaging of the heart. Circulation 2002;105:539-42.

6. Germano G, Kavanagh PB, Waechter P, et al. A new algorithm for the quantitation of myocardial perfusion SPECT. I: Technical principles and reproducibility. J Nucl Med 2000;41:712-9.

7. Slomka PJ, Nishina H, Berman DS, et al. Automated quantification of myocardial perfusion SPECT using simplified normal limits. J Nucl Cardiol 2005;12:66-77.

8. Sharir T, Germano G, Waechter PB, et al. A new algorithm for the quantification of myocardial perfusion SPECT. II: Validation and diagnostic yield. J Nucl Med 2000;41:720-7.

9. Flamen P, Bossuyt A, Franken PR. Technetium-99m-tetrofosmin Dipyridamole-stress myocardial SPECT imaging: Intraindividual comparison with technetium-99m-sestamibi. J Nucl Med 1995;36: 2009-15.

10. Slomka PJ, Berman DS, Germano G. Quantification of myocardial perfusion. In: Germano G, Berman DS, editors. Clinical gated cardiac SPECT. 2nd ed. Oxford: Blackwell; 2006. p. 69-91.

11. Costo S, Hugentobler A, Parienti J, Bouvard G, Grollier G, Agostini D. Usefulness of early post-stress tetrofosmin gated SPECT in comparison with delayed imaging to detect myocardial perfusion damages. Eur J Nucl Med Mod Imaging 2007;34:S224.

12. Guernou M, Manrique A, Benhabib $\mathrm{H}$, et al. Evaluation de la faisabilité de la tomographie myocardique précoce au sestamibi synchronisée à l'électrocardigramme. Méd Nucl 2007;31:647-55.

13. Georgoulias P, Demakopoulos N, Kontos A, Xaplanteris P, Xydis $\mathrm{K}$, Fezoylidis I. Early post-stress pulmonary uptake of $99 \mathrm{mTc}$ tetrofosmin during exercise (SPECT) myocardial perfusion imaging: Correlation with haemodynamic, perfusion and function parameters. Nucl Med Commun 2006;27:119-26.

14. Schulz G, Ostwald E, Kaiser HJ, vom Dahl J, Kleinhans E, Buell U. Cardiac stress-rest single-photon emission computed tomography with technetium 99m-labeled tetrofosmin: Influence of washout kinetics on regional myocardial uptake values of the rest study with a 1-day protocol. J Nucl Cardiol 1997;4:298-301.

15. Sinusas AJ, Shi QX, Saltzberg MT, Vitols P, Jain D, Wackers FJTh, et al. Technetium-99m-tetrofosmin to assess myocardial blood flow: Experimental validation in an intact canine model of ischemia. J Nucl Med 1994;35:664-71.

16. Ito Y, Uehara T, Fukuchi K, Tsujimura E, Hasegawa S, Nishimura T. Comparison of dual-isotope acquisition of $201 \mathrm{Tl}$ and $99 \mathrm{Tc}^{\mathrm{m}}$ tetrofosmin for the detection of ischaemic heart disease and determination of the optimal imaging time of $99 \mathrm{Tc}^{\mathrm{m}}$-tetrofosmin. Nucl Med Commun 1998;19:119-26.

17. Shaw LJ, Berman DS, Maron DJ, et al. Optimal medical therapy with or without percutaneous coronary intervention to reduce ischemic burden: Results from the clinical outcomes utilizing 
revascularization and aggressive drug evaluation (COURAGE) trial nuclear substudy. Circulation 2008;117:1283-91.

18. Hansen CL, Goldstein RA, Akinboboye OO, et al. ASNC imaging guidelines for nuclear cardiology procedures-myocardial perfusion and function: Single photon emission tomography. J Nucl Cardiol 2007;14e:39-60.

19. Emmett L, Iwanochko RM, Freeman MR, Barolet A, Lee DS, Husain M. Reversible regional wall motion abnormalities on exercise technetium-99m-gated cardiac single photon emission computed tomography predict high-grade angiographic stenoses. J Am Coll Cardiol 2002;39:991-8.

20. Parker Ward R, Gundeck EL, Lang RM, Spencer KT, Williams KA. Overestimation of postischemic myocardial stunning on gated SPECT imaging: Correlation with echocardiography. J Nucl Cardiol 2006;13:514-20.

21. Bonow RO. Gated myocardial perfusion imaging for measuring left ventricular function. J Am Coll Cardiol 1997;30:1649-50.

22. Toba M, Kumita S, Cho K, Ibuki C, Kumazaki T, Takano T. Usefulness of gated myocardial perfusion SPECT imaging soon after exercise to indentify postexercise stunning in patients with single-vessel coronary artery disease. J Nucl Cardiol 2004;11:697703.

23. Paul AK, Hasegawa S, Yoshioka J, et al. Characteristics of regional myocardial stunning after exercise in gated myocardial SPECT. J Nucl Cardiol 2002;9:388-94.

24. Reyes E, Loong CY, Harbinson M, et al. A comparison of Tl-201, Tc-99m sestamibi, and Tc-99m tetrofosmin myocardial perfusion scintigraphy in patients with mild to moderate coronary stenosis. J Nucl Cardiol 2006;13:488-94.

25. Acampa W, Cuocolo A, Sullo P, et al. Direct comparison of technetium-99m-sestamibi and technetium 99m-tetrofosmin cardiac single photon emission computed tomography in patients with coronary artery disease. J Nucl Cardiol 1998;5:265-74.

26. Berman DS, Abidov A, Kang X, et al. Prognostic validation of a 17-segment score derived from a 20-segment score for myocardial perfusion SPECT interpretation. J Nucl Cardiol 2004;11:414-23. 